\title{
Trim47 overexpression correlates with poor prognosis in gastric cancer
}

\author{
YuJian XIA ${ }^{1, *}$, ZheWei WEI ${ }^{1, *}$, WeiBin HUANG ${ }^{1}$, XiaoJing WEI'², YuLong $\mathrm{HE}^{1, *}$ \\ ${ }^{1}$ Department of Gastrointestinal Surgery, The First Affiliated Hospital of Sun Yat-sen University, Guangzhou, Guangdong, China; ${ }^{2}$ Department \\ of Medical Record Management, Affiliated Hospital of Yangzhou University, Yangzhou, Jiangsu, China \\ ${ }^{*}$ Correspondence: heyulong@mail.sysu.edu.cn \\ \#Contributed equally to this work.
}

Received July 8, 2020 / Accepted October 29, 2020

\begin{abstract}
Trim47 is a member of the tripartite motif (TRIM) family that participates in many pathophysiological processes. However, the expression pattern and biological functions of Trim 47 in gastric cancer (GC) remain unclear. The present study aimed to further explore the clinicopathological significance and potential prognostic role of Trim47 expression in GC. Therefore, in this study, Trim 47 mRNA level was investigated in the Cancer Genome Atlas (TCGA) and Oncomine database in GC. We detected Trim 47 mRNA and protein expression levels in GC and paired adjacent normal tissues. Kaplan-Meier method and Cox proportional hazard regression models were performed to analyze the survival of patients and prognostic factors. A gene set enrichment analysis (GSEA) was performed to determine the mechanism of Trim47 in GC. Our results indicated that Trim 47 mRNA expression in GC tissues was significantly higher than adjacent normal tissues, as was Trim47 protein expression. Trim47 overexpression in GC tissues was significantly associated with tumor differentiation, T stage, $\mathrm{N}$ stage, M stage, and TNM stage. Kaplan-Meier analyses showed that high Trim47 expression was associated with worse overall survival (OS) and disease-free survival (DFS) in GC patients. Multivariate analysis confirmed that Trim47 expression was an independent prognostic factor for GC patients. Bioinformatics analysis and western blot indicated Trim47 might regulate GC through NF- $\mathrm{B}$, EMT, hypoxia, and apoptosis signaling pathway in GC. Our results show that Trim 47 has the potential to serve as a novel prognostic biomarker in GC patients.
\end{abstract}

Key words: Trim47, gastric cancer, prognosis, immunohistochemistry, overall survival

Among cancers, gastric cancer (GC) has become a common malignant tumor in humans and a major health problem. GC ranks sixth for cancer incidence and third for cancer deaths with 834,000 deaths $[1,2]$. Surgical resection is a curative treatment for early-diagnosed GC patients, but many patients are diagnosed at inoperable advanced stages or suffer from metastasis after resection [3-5]. The 5-year overall survival rate of GC patients is still very low due to the majority of patients already being at a progressive stage when diagnosed [6-8]. Therefore, there is an urgent need to identify novel biomarkers for early diagnosis, prediction of metastatic progression, and prognosis of GC patients.

Tripartite motif (TRIM) family proteins are members of the RING family of ubiquitin E3 ligases, which are characterized by three conserved domains, composed of RING, B-BOX, and coiled-coil (RBCC) [9-11]. TRIM family proteins participate in a wide array of cellular activities, such as cell cycle regulation, cell proliferation, migration, differentiation, apoptosis, inflammation, carcinogenesis, and immunity [12-19]. Trim47, a member of the TRIM family proteins, is localized to $17 \mathrm{q} 24-25$, a region that is frequently gained or amplified in multiple human tumor types [20]. Therefore, Trim 47 may also be associated with the progression and prognosis of human tumors. In addition, Trim47 expression has been confirmed as a predictor of survival in patients with prostate cancer, non-small cell lung cancer, colorectal cancer, and breast cancer [21-24]. Nonetheless, it remains unclear whether Trim 47 regulates the progression and prognosis of GC.

Therefore, the present study was conducted to investigate the expression of Trim47 in GC tissues and analyze its association with patient's clinical significance and prognosis.

\section{Materials and methods}

Clinical specimens. Clinical gastric cancer samples were obtained from the First Affiliated Hospital of Sun Yat-sen University between 2004 and 2008. 136 patients 
with primary GC who had undergone gastric resection with lymph node dissection were randomly selected for this study. No patients received preoperative chemotherapy or radiation therapy. The diagnosis of GC was histologically confirmed by a pathologist. The stage of GC was determined according to the 8th edition of the tumor-node-metastasis (TNM) classification of malignant tumors established by the American Joint Committee on Cancer (AJCC) [25]. Another 30 GC tissues and paired adjacent normal tissues were obtained randomly by surgery from GC patients in 2019. The study was approved by the Ethical Review Committee of the First Affiliated Hospital of Sun Yat-sen University.

Cell culture, reagents, and transfection. The human GC cell line (AGS) was purchased from the Cell Bank of the Chinese Academy of Science (Shanghai, China). The cell line was cultured in RPMI-1640 medium (Gibco, Grand Island, NY, USA) supplemented with $10 \%$ fetal bovine serum (Gibco, Grand Island, NY, USA), appropriate amounts of penicillin $(100 \mathrm{U} / \mathrm{ml})$ and streptomycin $(100 \mu \mathrm{g} / \mathrm{ml})$, at $37^{\circ} \mathrm{C}$ in air containing $5 \% \mathrm{CO}_{2}$. Antibodies against Trim 47 (\#26885-1-AP), HIF1a (\#20960-1-AP), Vimentin (\#603301-lg), E-Cadherin (\#20874-1-AP), and GAPDH (\#600041-lg) were purchased from Proteintech Group (Wuhan, China). Antibody against Bcl2 (\#ab32124) was purchased from Abcam (Cambridge, MA, USA). Antibodies against N-Cadherin (\#13116), P65 (\#8242), P-P65 (\#3033), and Caspase-3 (Cleaved-Caspase-3) (\#9662) were purchased from Cell Signaling Technology (Danvers, MA, USA).

The Trim47 siRNA and negative control siRNA (siNC) were purchased from RiboBio (Guangzhou, China). Cells were transfected with Trim 47 siRNA or negative control siRNA using Lipofectamine 3000 according to the manufacturer's instructions.

Quantitative real-time PCR (qRT-PCR). Total RNA was extracted using TRIzol reagent (Invitrogen) and reversely transcribed to cDNA following the manufacturer's instructions. Primers for qRT-PCR were used as follows: Trim47 forward primer 5'-GCTTCAGGAGGCTGAGCAGT-3', reverse primer 5'-TCTGCTACGGCTGCACTCTT-3', GAPDH forward primer 5'-ATGTTGCAACCGGGAAGGAA-3', reverse primer 5'-AGGAAAAGCATCACCCGGAG-3'. The SYBR Premix Ex Taq ${ }^{\text {ma }}$ II and CFX96 Touch ${ }^{\text {mo }}$ Real-Time PCR Detection System (Bio-Rad, CA, USA) were used for qPCR assays.

Western blot analysis. Total protein was extracted from tissues or cells. Protein samples were separated by SDS-PAGE and transferred to PVDF membranes (Millipore, Billerica, MA, USA). After blocking with $5 \%$ skim milk in TBST, membranes were incubated with specific primary antibodies overnight at $4{ }^{\circ} \mathrm{C}$. Subsequently, the membranes were incubated with HRP-conjugated secondary antibody. Protein bands were detected with the enhanced chemiluminescence reagent (Millipore, Billerica, MA, USA).

Immunohistochemistry (IHC) staining. The consecutive sections $4 \mu \mathrm{m}$ in thickness were cut from each tissue-array block and used for IHC staining. The sections were deparaffinized, rehydrated, and boiled in a microwave oven with citrate buffer ( $\mathrm{pH}$ 6.0) for $8 \mathrm{~min}$ for antigen retrieval. Endogenous peroxidase activity was blocked by incubating the slides in hydrogen peroxide (3\%). The sections were blocked with normal goat serum to block nonspecific binding. Then sections were incubated with anti-Trim 47 antibody (1:100, Proteintech, 26885-1-AP) overnight at $4^{\circ} \mathrm{C}$. Subsequently, sections were incubated with an HRP-conjugated sheep anti-rabbit secondary antibody (DakoCytomation, Glostrup, Denmark) and diaminobenzidine (DAB). Finally, the sections were counterstained with hematoxylin, dehydrated, and mounted.

The result of the staining of Trim 47 for each section was assessed independently by two authors in a blinded manner according to the semiquantitative scoring system. First, no staining or staining of less than $10 \%$ of the tumor cells was considered negative (IHC score 0). Second, for more than $10 \%$ of the tumor cells, IHC was scored using the intensity of staining ( 1 , weak staining; 2 , moderate staining; 3 , strong staining). Trim 47 expression was finally classified into low $(0$, $1)$ and high $(2,3)$.

Bioinformatics analysis. The RNAseq data of GC was downloaded from the Cancer Genome Atlas (TCGA) (https://genomecancer.ucsc.edu) and Oncomine databases (https://www.oncomine.org). GSEA v4.0.3 software was used to perform GSEA and analyze differences in the biologic pathway. Trim 47 mRNA expression level was separated into a high and a low group (critical value $=$ median Trim 47 level). Functional gene sets were defined according to Molecular Signatures Database v7.1 (MSIGDB). A p $<0.05$ and FDR $<0.25$ were recognized as differentially expressed threshold.

Statistical analyses. Statistical analyses were performed using SPSS 22.0 (IBM Co, Chicago, IL, USA) and GraphPad Prism software 5.0 (GraphPad software, La Jolla, CA, USA). All data were presented as the mean \pm SD. Student's t-test was used to determine the statistical differences between different groups. The relationships between Trim 47 expression and clinicopathological characteristics were compared by $\chi^{2}$ test or Fisher exact test (two-sided). Survival curves were constructed using the Kaplan-Meier method, and differences between survival curves were determined to be significant based on the log-rank test. The Cox regression model analysis was performed to identify the independent prognostic factors of survival. A p-value $<0.05$ was considered statistically significant.

\section{Results}

Overexpression of Trim47 in GC tissues. We analyzed TCGA databases through UALCAN [26] and found that the Trim 47 gene was upregulated in GC tissues compared with normal tissues (Figure 1A). The Trim 47 mRNA level in GC tissues $(n=415)$ and normal tissues $(n=34)$ from the TCGA cohort showed that Trim 47 mRNA expression was 
significantly upregulated in GC tissues ( $p<0.001$; Figure $1 \mathrm{~B})$. Compared with normal tissue, the Trim 47 mRNA level was also upregulated with different tumor stage $(\mathrm{p}<0.001$; Figure $1 \mathrm{C})$. Similar results were observed in other GC cohorts from the Oncomine database [27-30] (Figure 1D; Table 1). We detected Trim47 mRNA expression level in 21 GC and paired adjacent normal tissues by qRT-PCR (Figure 1E) and protein expression level in GC and paired adjacent normal tissues by western blot (Figure $1 \mathrm{~F}$ ) and IHC staining (Figure 2). The results showed that Trim 47 mRNA and protein expres-
A

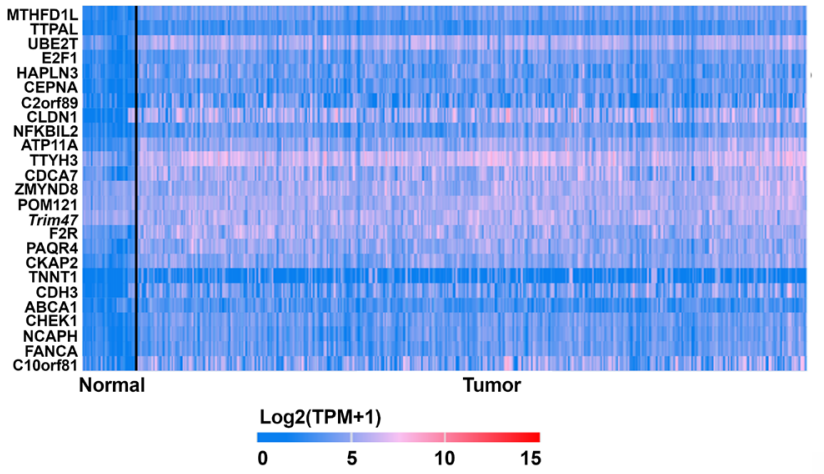

C

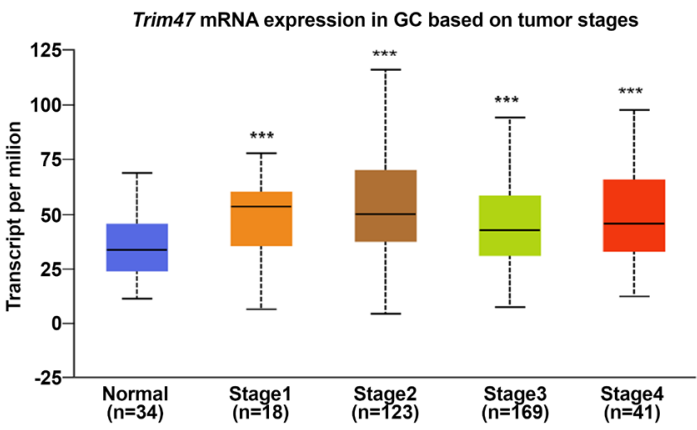

$\mathbf{E}$

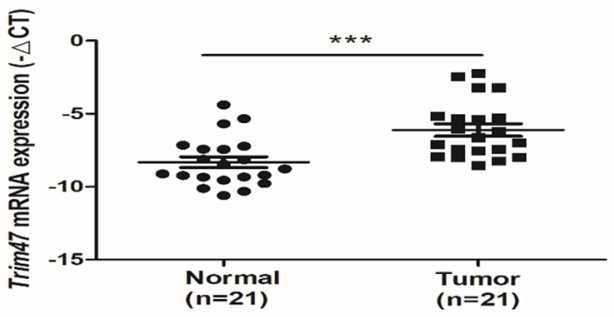

B

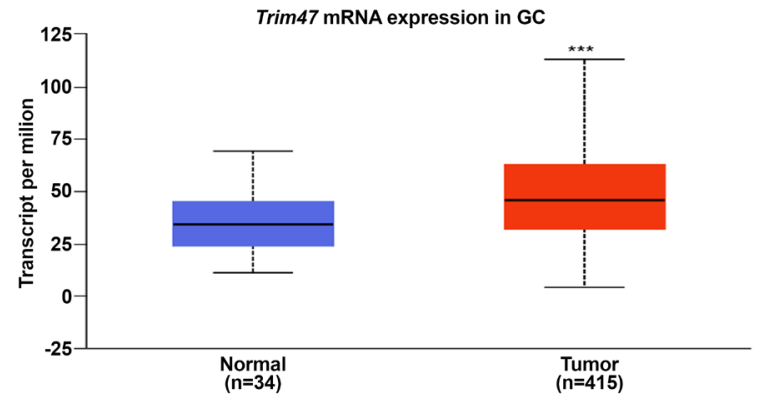

D

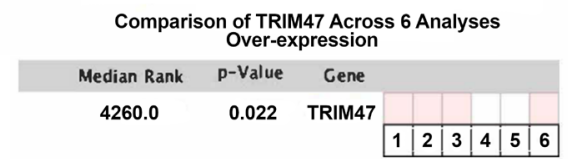

Legend

1. Gastric Mixed Adenocarcinoma vs.

Cho Gastric. Clin Cancer Res. 2011

2. Gastric Cancer vs. Normal

Cui; Gastric, Nucleic Acids Res, 201

3. Diffuse Gastric Adenocarcinoma vs.

Normal

DErrico Gastric, Eur J Cancer, 200

$\begin{array}{llllll}1 & 5 & 1025 & 25105 & 1\end{array}$

$\square \square \square \square \square \square \square \square \square$ Not measured

The rank for gene is the median rank for that gene across each of the analyses.
The $p$-value for a gene is its $p$-value for the median-ranked analysis.

$\mathbf{F}$

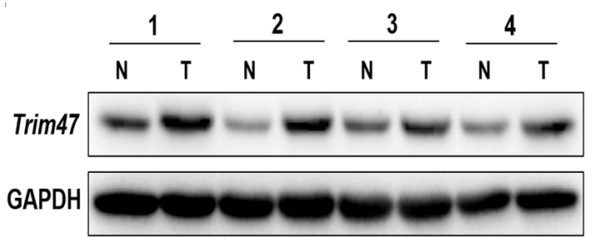

Figure 1. Increased Trim 47 expression in gastric cancer. A) Analysis of the TCGA data by a heat map. B) Trim $47 \mathrm{mRNA}$ expression in GC and normal tissues from the TCGA database. C) Trim47 mRNA expression based on tumor stage from the TCGA database. D) Six analyses were performed on the Oncomine database in comparing mRNA expression of Trim 47 between GC and normal tissues. E) Detection of Trim $47 \mathrm{mRNA}$ expression level in GC and paired adjacent normal tissues by qRT-PCR. F) Trim 47 protein expression level was measured in GC and paired adjacent normal tissues by a western blot. The data are represented as mean $\pm \mathrm{SD}$. ${ }^{\star * *} \mathrm{p}<0.001$

Table 1. Oncomine analysis of Trim 47 expression in gastric cancer (total of 4 GC cohorts).

\begin{tabular}{|c|c|c|c|c|}
\hline Cohort & Sample (n) & t-test & Fold change & p-value \\
\hline \multirow[t]{3}{*}{ D’Errico et al. [30] } & diffuse gastric adenocarcinoma (6) vs. normal (31) & 2.387 & 1.585 & 0.013 \\
\hline & gastric mixed adenocarcinoma (4) vs. normal (31) & 2.283 & 1.852 & 0.032 \\
\hline & gastric intestinal type adenocarcinoma (26) vs. normal (31) & 2.232 & 1.668 & 0.015 \\
\hline Cui et al. [28] & gastric cancer (80) vs. normal (80) & 1.747 & 1.216 & 0.041 \\
\hline Wang et al. [27] & gastric cancer (12) vs. normal (15) & 2.221 & 1.459 & 0.018 \\
\hline Cho et al. [29] & gastric mixed adenocarcinoma (10) vs. normal (19) & 2.043 & 1.372 & 0.026 \\
\hline
\end{tabular}


sion levels were significantly higher in GC compared with adjacent normal tissues. Taken together, the above data showed that Trim 47 was upregulated in GC tissues.

Correlation between Trim47 expression and clinicopathological characteristics in GC. To explore the association between Trim 47 expression and clinicopathological characteristics in GC patients, we performed IHC staining to detect Trim 47 expression in 136 GC specimens. As shown in Figure 2A, Trim 47 protein was mainly distributed in the cytoplasm. The relationships between Trim 47 expression and clinicopathological characteristics are summarized in Table 2. Trim 47 overexpression in GC tissues was significantly related to tumor differentiation $(\mathrm{p}=0.021)$, $\mathrm{T}$ stage $(\mathrm{p}=0.010), \mathrm{N}$ stage $(\mathrm{p}=0.003), \mathrm{M}$ stage $(\mathrm{p}=0.016)$, and TNM stage $(\mathrm{p}=0.001)$ (Table 2$)$. Nevertheless, other clinical characteristics such as gender, age, tumor size, and CEA level were not associated with the expression of Trim 47.
Trim47 overexpression indicated a worse prognosis in GC patients. The follow-up period of the 136 GC patients ranged from 1.7 to 152.5 months. The 5-year OS rate was $79.4 \%$ in the low Trim 47 expression group and $31.6 \%$ in the high Trim 47 expression group. Our data showed that high Trim47 expression was associated with worse OS $(\mathrm{p}<0.001$; Figure $3 \mathrm{~A})$ and DFS $(\mathrm{p}<0.001$; Figure $3 \mathrm{~B})$ in GC patients. Furthermore, we also detected the prognostic value of Trim47 expression in early (TNM stage I and II) and advanced (TNM stage III and IV) GC. The results indicated that high Trim 47 expression was associated with worse OS $(p<0.05$, Figures $3 C, 3 E)$ and DFS $(p<0.05$, Figures $3 D, 3 F)$ in both early and advanced GC.

To determine whether Trim47 expression was an independent prognostic predictor for patients with GC, we performed univariate Cox regression analysis to assess the impact of Trim47 expression level and other clinicopatho-
A

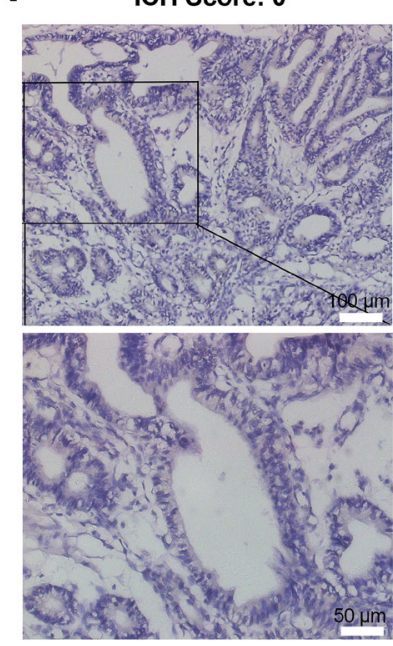

B
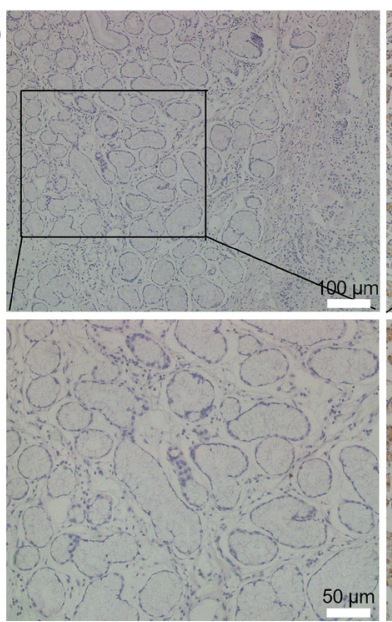

ICH Score: 1
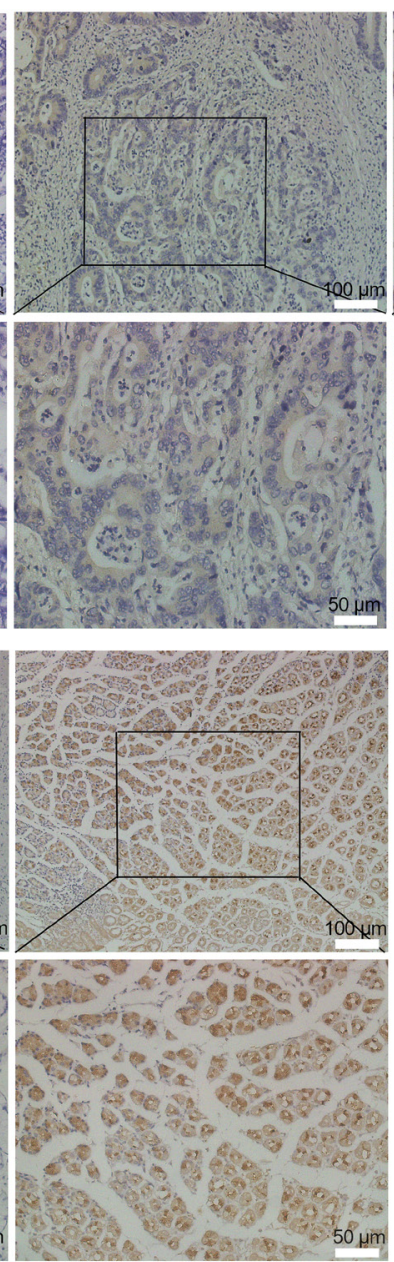

ICH Score: 2
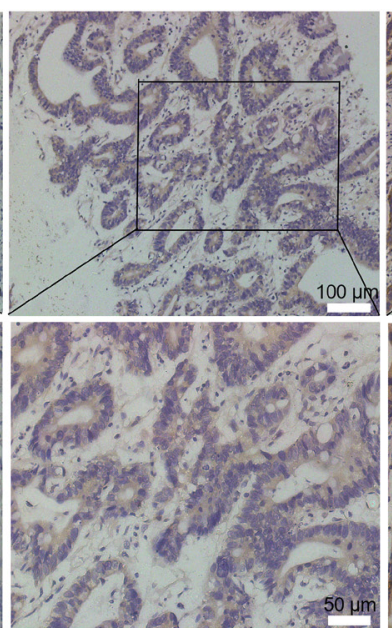

C

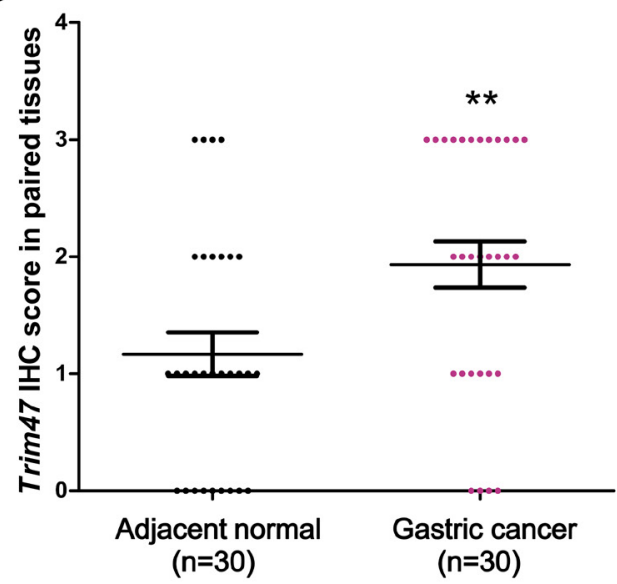

Figure 2. Detection of Trim47 protein expression in GC tissues and adjacent normal tissues by IHC staining. A) IHC staining of Trim47 protein in GC tissues. IHC scoring was performed according to the staining intensity ( 0 , negative; 1 , weak; 2 , moderated; 3 , strong). B) IHC staining of Trim47 protein in adjacent normal tissues (left panel, negative; right panel, positive). C) Protein expression of Trim47 was significantly higher in GC tissues compared with adjacent normal tissues by IHC (original views $\times 100$, enlarged views $\times 200$ ). The data are presented as mean \pm SD. ${ }^{* *} \mathbf{p}<0.01$ 
Table 2. Relationship between Trim 47 expression levels and clinicopathological characteristics in GC patients.

\begin{tabular}{|c|c|c|c|c|c|}
\hline \multirow{2}{*}{ Characteristics } & \multirow{2}{*}{$\mathrm{N}(136)$} & \multicolumn{2}{|c|}{ Trim47 expression } & \multirow{2}{*}{$\chi^{2}$} & \multirow{2}{*}{ p-value } \\
\hline & & Low (44) & High (92) & & \\
\hline \multicolumn{6}{|l|}{ Gender } \\
\hline Male & 92 & 32 & 60 & 0767 & 0.381 \\
\hline Female & 44 & 12 & 32 & & \\
\hline \multicolumn{6}{|l|}{ Age (years) } \\
\hline$\leq 60$ & 75 & 24 & 51 & 0.010 & 0.922 \\
\hline$>60$ & 61 & 20 & 41 & & \\
\hline \multicolumn{6}{|l|}{ Tumor size (cm) } \\
\hline$\leq 4$ & 88 & 30 & 58 & 0.344 & 0.557 \\
\hline$>4$ & 48 & 14 & 34 & & \\
\hline \multicolumn{6}{|l|}{ Tumor differentiation } \\
\hline Well or moderate & 58 & 25 & 33 & 5.340 & 0.021 \\
\hline Poor & 78 & 19 & 59 & & \\
\hline \multicolumn{6}{|l|}{ T stage } \\
\hline $\mathrm{T} 1 / \mathrm{T} 2$ & 58 & 25 & 31 & 6.570 & 0.010 \\
\hline $\mathrm{T} 3 / \mathrm{T} 4$ & 80 & 19 & 61 & & \\
\hline \multicolumn{6}{|l|}{$\mathrm{N}$ stage } \\
\hline No & 42 & 21 & 21 & 8.647 & 0.003 \\
\hline $\mathrm{N} 1 / \mathrm{N} 2 / \mathrm{N} 3$ & 94 & 23 & 71 & & \\
\hline \multicolumn{6}{|l|}{ M stage } \\
\hline M0 & 111 & 41 & 70 & 5.798 & 0.016 \\
\hline M1 & 25 & 3 & 22 & & \\
\hline \multicolumn{6}{|l|}{ TNM stage } \\
\hline $\mathrm{I} / \mathrm{II}$ & 50 & 25 & 25 & 11.251 & 0.001 \\
\hline III/IV & 86 & 19 & 67 & & \\
\hline \multicolumn{6}{|l|}{ CEA level $(\mu \mathrm{g} / \mathrm{l})$} \\
\hline$<5$ & 110 & 38 & 72 & 1.264 & 0.261 \\
\hline$\geq 5$ & 26 & 6 & 20 & & \\
\hline
\end{tabular}

Table 3. Univariate and multivariate Cox regression analysis for OS.

\begin{tabular}{lccccc}
\hline \multirow{2}{*}{ Characteristics } & \multicolumn{2}{c}{ Univariate analysis } & & \multicolumn{2}{c}{ Multivariate analysis } \\
\cline { 2 - 3 } \cline { 5 - 6 } Gender & HR (95\%CI) & p-value & & HR (95\%CI) & p-value \\
Age & $0.683(0.430-1.084)$ & 0.106 & & \\
Tumor size & $1.123(0.717-1.758)$ & 0.613 & & \\
Tumor differentiation & $1.376(0.874-2.166)$ & 0.169 & & \\
T stage & $1.575(0.990-2.505)$ & 0.055 & & & \\
N stage & $2.440(1.471-4.048)$ & $\mathbf{0 . 0 0 1}$ & & & \\
M stage & $2.184(1.291-3.695)$ & $\mathbf{0 . 0 0 4}$ & & & \\
TNM stage & $6.678(3.838-11.620)$ & $<\mathbf{0 . 0 0 1}$ & & $3.407(1.886-6.154)$ & $<\mathbf{0 . 0 0 1}$ \\
CEA concentration & $3.172(1.878-5.356)$ & $<\mathbf{0 . 0 0 1}$ & & $1.823(1.019-3.261)$ & $\mathbf{0 . 0 4 3}$ \\
Trim47 expression & $1.419(0.825-2.440)$ & 0.207 & & & \\
\hline
\end{tabular}

logical factors on the prognosis of GC patients. Univariate analysis revealed that clinical variables including $\mathrm{T}$ stage, $\mathrm{N}$ stage, $\mathrm{M}$ stage, TNM stage, and Trim47 expression were significantly associated with OS (Table 3). Furthermore, multivariate Cox analysis showed that Trim 47 expression was an independent predictor of OS in GC patients $(\mathrm{HR}=2.927$, 95\% CI: 1.559-5.494, $\mathrm{p}=0.001$, Table 3). Moreover, Trim 47 expression was also an independent predictor of DFS in GC patients (HR=3.382, 95\% CI: 1.795-6.373, p<0.001, Table 4). These results indicated that high Trim 47 expression was an independent prognostic predictor for GC patients.

The main enriched pathways in GC tissues with high Trim47 expression. GSEA is a microarray data analysis method that uses predefined gene sets and ranks of genes to identify more subtle changes of gene expression $[31,32]$. In this study, to explore the role of Trim 47 in GC prolifera- 
A

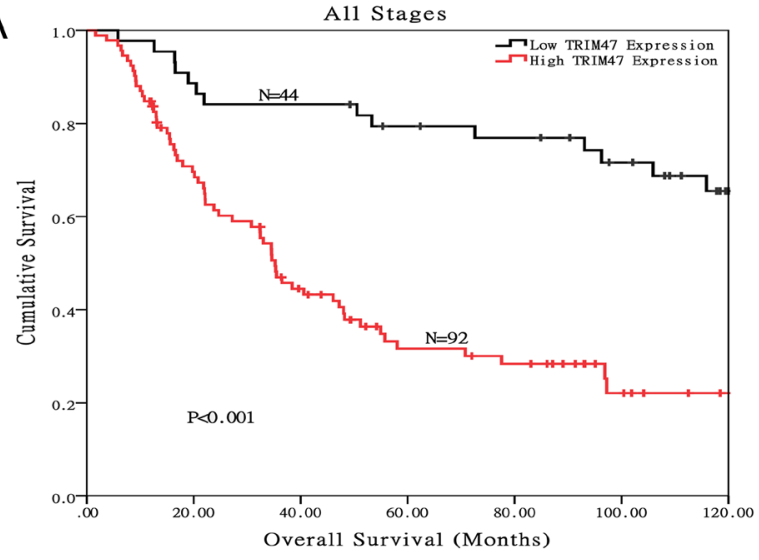

C

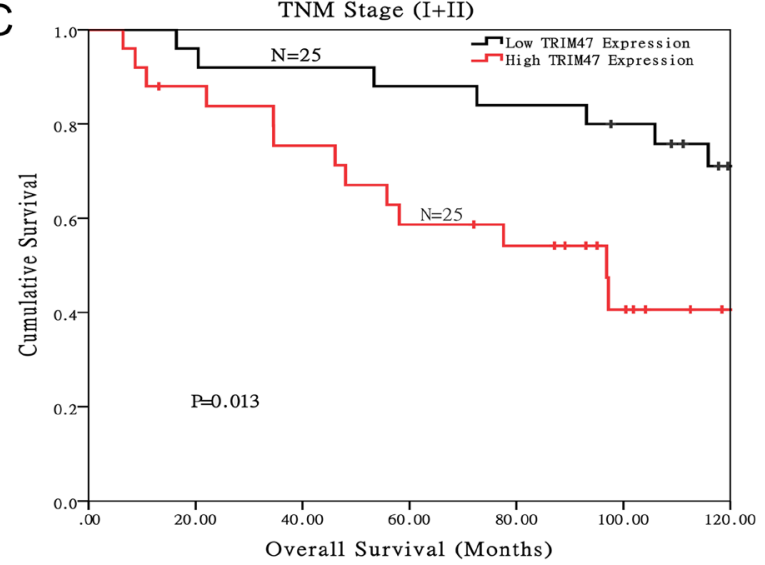

$E$

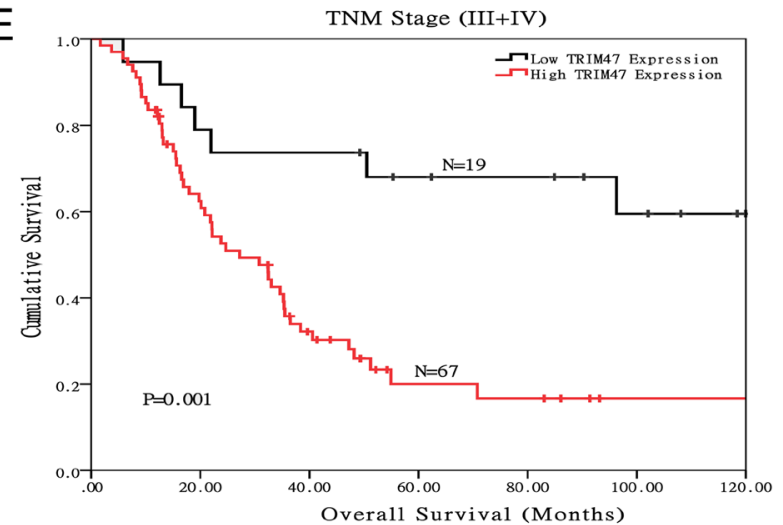

B TNM Stage (I+II+III)

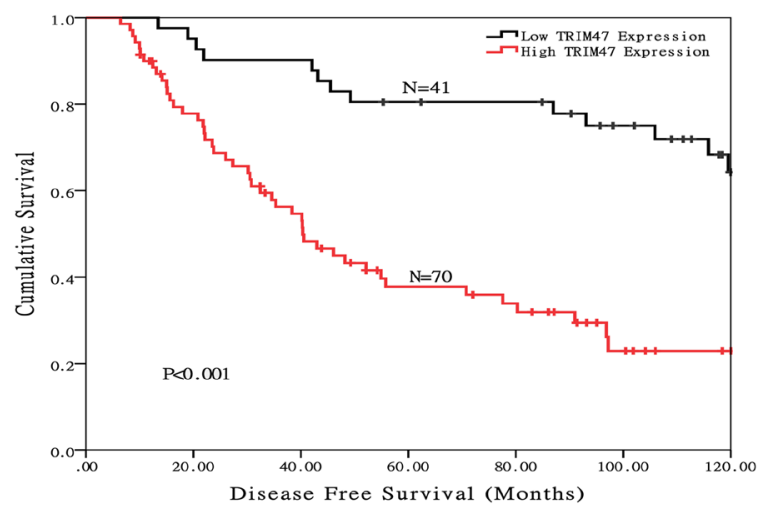

D

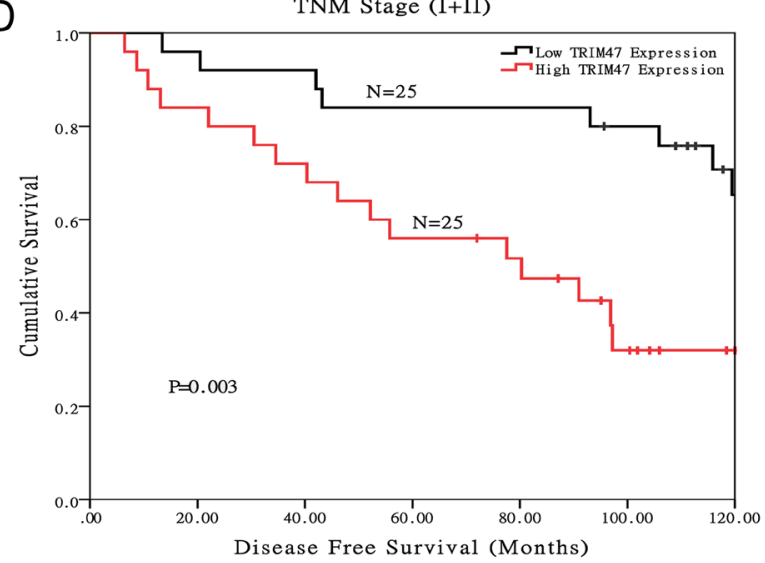

F

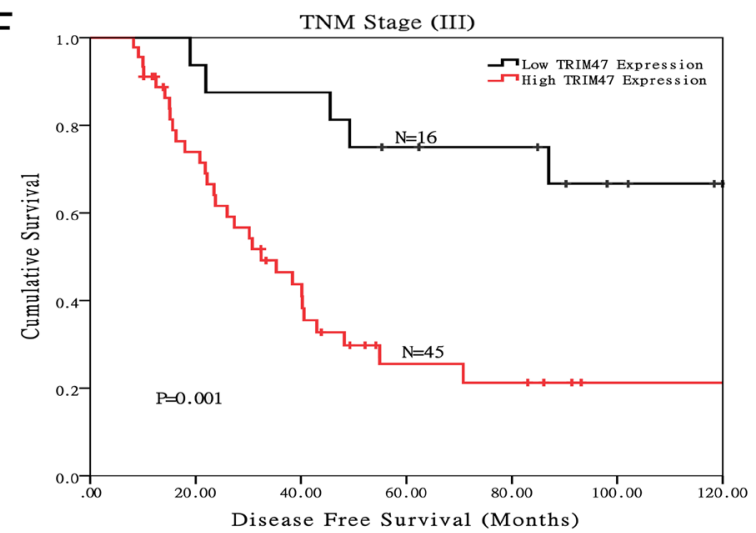

Figure 3. High Trim47 expression in tumors predicted poor prognosis in GC patient. GC patients with high Trim47 expression had worse OS A) and DFS B) than those with low Trim47 expression. Patients with high Trim47 expression had worse OS C) and DFS D) in early GC. Patients with high Trim 47 expression had a worse OS (E) and DFS (F) in advanced GC.

tion and metastasis, an integrative analysis of GC microarray was carried out on the base of the TCGA database. The GSEA results showed that the high Trim47 expression group was significantly enriched in NF- $\kappa \mathrm{B}$, epithelial-tomesenchymal transition (EMT), hypoxia, and apoptosis signaling pathway (Figures 4A-4D). We further confirmed that Trim 47 regulated GC through NF- $\mathrm{kB}$, EMT, hypoxia, and apoptosis signaling pathway by western blot. We established Trim 47 knockdown in AGS cells to ascertain the roles of Trim47 in signaling pathway (Figure 4E). As to EMT, we performed a western blot to measure the expression of EMT markers in our cell model. Then we found that the epithelial marker E-cadherin was elevated significantly in Trim 47 knockdown cells, while the mesenchymal markers 
A

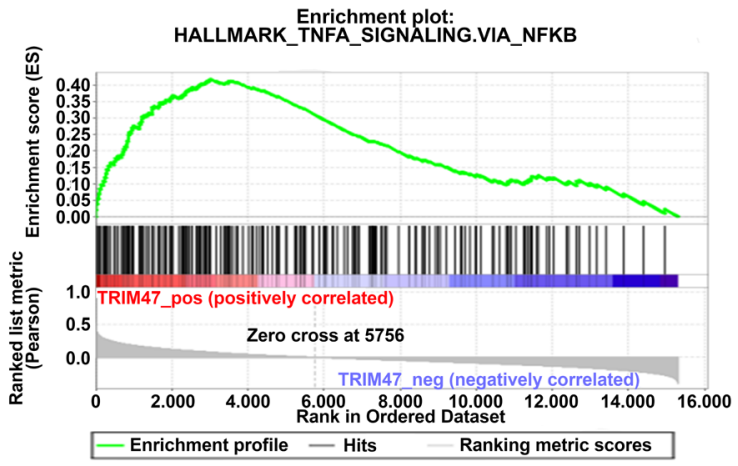

C

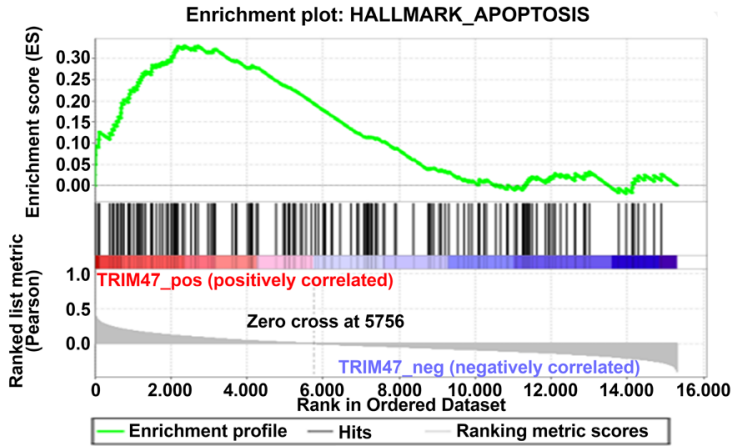

B

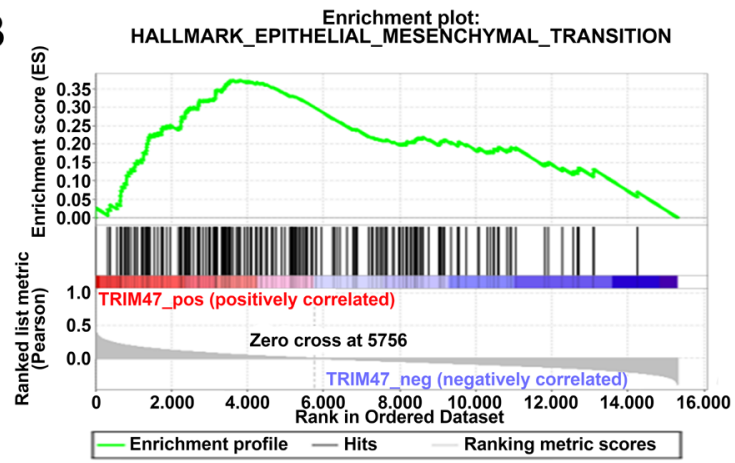

D

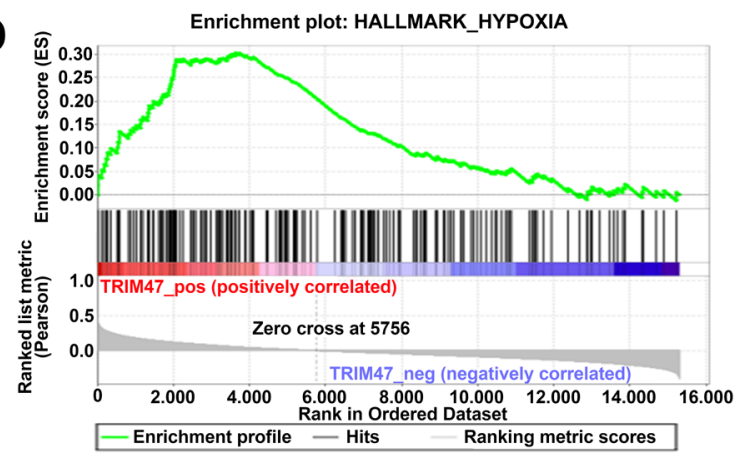

F
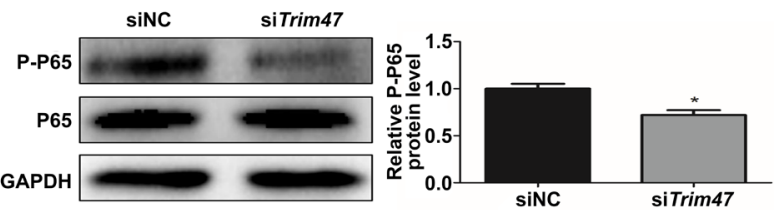

G

E

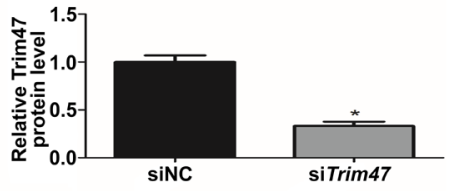

GAPDH
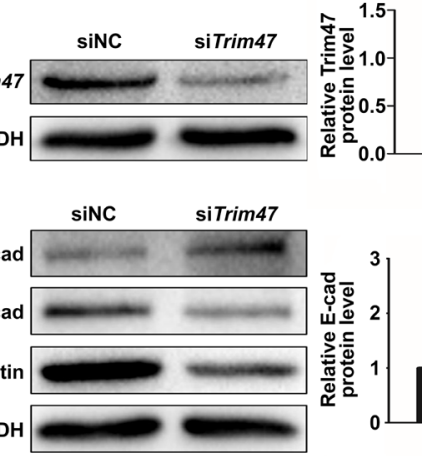

siNC

siTrim47
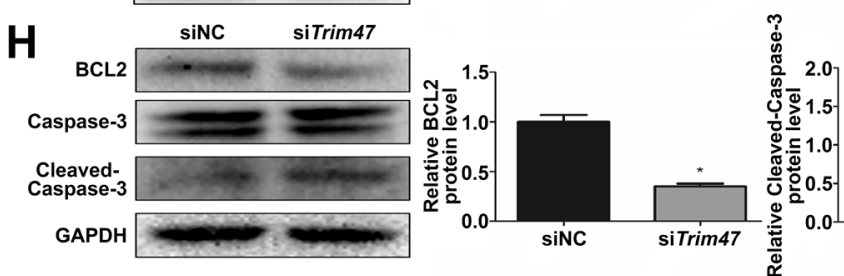
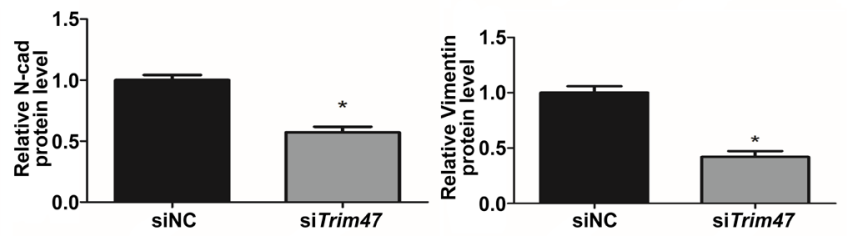

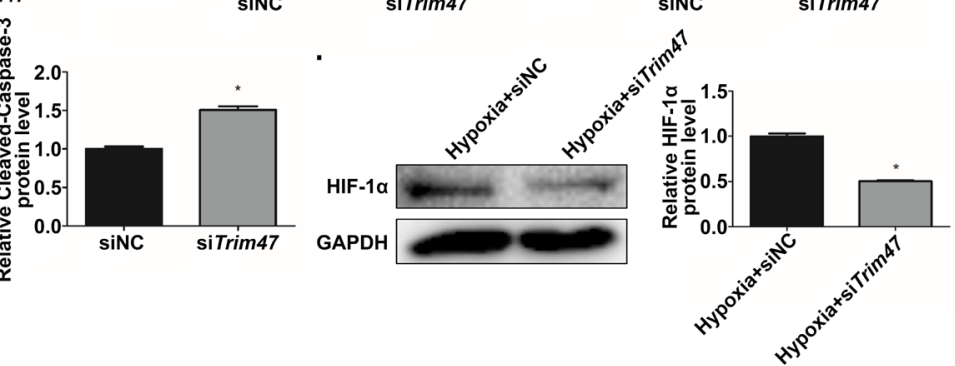

Figure 4. The main enriched pathways. A) High expression of Trim47 was enriched in the NF- $\mathrm{kB}$ pathway by GSEA analysis. B) High expression of Trim 47 was enriched in the EMT pathway by GSEA analysis. C) High expression of Trim47 was enriched in the apoptosis pathway by GSEA analysis. D) High expression of Trim47 was enriched in the hypoxia pathway by GSEA analysis. E) The effect of Trim47 knockdown level was confirmed by western blot analysis in AGS cells. F) Western blot analysis of P-P65 and P65 in Trim47 knockdown and control AGS cells. G) Western blot analysis of Ecadherin, N-cadherin, and Vimentin in Trim47 knockdown and control AGS cells. H) Western blot analysis of Bcl2, Caspase-3, and cleaved Caspase-3 in Trim47 knockdown and control AGS cells. I) Western blot analysis of HIF1a in Trim47 knockdown and control AGS cells in hypoxic conditions. The data are presented as mean $\pm \mathrm{SD} .{ }^{*} \mathrm{p}<0.05$ 
Table 4. Univariate and multivariate Cox regression analysis for DFS.

\begin{tabular}{lccccc}
\hline \multirow{2}{*}{ Characteristics } & \multicolumn{2}{c}{ Univariate analysis } & & \multicolumn{2}{c}{ Multivariate analysis } \\
\cline { 2 - 3 } \cline { 5 - 6 } Gender & $0.684(0.409-1.144)$ & p-value & & HR (95\%CI) & p-value \\
Age & $1.244(0.757-2.046)$ & 0.148 & & & $\mathbf{0 . 0 2 3}$ \\
Tumor size & $1.641(0.996-2.704)$ & 0.052 & & & 0.018 \\
Tumor differentiation & $1.795(1.071-3.006)$ & $\mathbf{0 . 0 2 6}$ & & $1.841(1.088-3.115)$ & \\
T stage & $2.278(1.336-3.885)$ & $\mathbf{0 . 0 0 3}$ & & $1.958(1.123-3.416)$ & \\
N stage & $1.769(1.031-3.034)$ & $\mathbf{0 . 0 3 8}$ & & & \\
TNM stage & $1.994(1.193-3.333)$ & $\mathbf{0 . 0 0 8}$ & & & \\
CEA concentration & $1.513(0.818-2.797)$ & 0.187 & & & \\
Trim47 expression & $4.086(2.222-7.513)$ & $<\mathbf{0 . 0 0 1}$ & & $3.382(1.795-6.373)$ & $\mathbf{0 . 0 0 1}$ \\
\hline
\end{tabular}

$\mathrm{N}$-cadherin and Vimentin were downregulated. The result showed that Trim47 knockdown inhibited the GC cell EMT process. We further confirmed the effect of Trim 47 on $\mathrm{NF}-\kappa \mathrm{B}$, hypoxia, and apoptosis signaling pathway in GC. The results suggested that Trim47 knockdown decreased P-P65, Bcl2, and HIF1a levels and promoted the cleaved Caspase- 3 level. The findings indicated that the expression of Trim47 was significantly associated with NF- $\mathrm{kB}, \mathrm{EMT}$, hypoxia, and apoptosis signaling pathway.

\section{Discussion}

TRAM proteins often form a medium-sized subfamily of mainly post-translational modification modifiers, including ubiquitylation and other ubiquitin-like modifications [33, 34]. Therefore, TRIM proteins are involved in inflammatory and innate immune responses, especially in carcinogenesis and progression of various tumors [13, 35]. Increasing evidence had demonstrated that Trim 47 was upregulated in tumor tissue and might serve as a new prognostic marker in patients with malignant tumors [21-24]. Fujimura et al. found that a higher Trim47 expression in prostate cancer tissues was significantly correlated with advanced pathologic T stage and worse cancer-specific survival rates [21]. Similarly, elevated Trim47 expression was also associated with tumor progression in breast cancer, non-small cell lung carcinoma, and colorectal cancer [22-24]. However, there was no study to explore the relationship between Trim47 expression and prognosis of GC.

In the present study, we identified that Trim 47 could be a potential new biomarker to diagnose and evaluate the prognosis of GC patients. First, we analyzed the data from TCGA and Oncomine database to prove that the level of Trim47 mRNA in GC tissues was significantly higher than that in normal tissues. We further confirmed the above result by $\mathrm{qRT}$-PCR in GC tissues and paired adjacent normal tissues. Second, we further assessed Trim 47 protein expression by western blot and IHC staining in GC tissues and paired adjacent normal tissues. The protein expression of Trim47 was significantly higher in GC compared with adjacent normal tissues. Third, we performed IHC staining and analyzed the relationship between Trim47 expression and prognosis in $136 \mathrm{GC}$ patients. The result showed that higher Trim47 expression was associated with worse OS and DFS in GC patients. Finally, the multivariate Cox regression analysis demonstrated that Trim47 expression was an independent predictor of OS and DFS in GC patients.

The findings of this study also revealed that Trim47 overexpression was significantly related to tumor differentiation, $\mathrm{T}$ stage, $\mathrm{N}$ stage, $\mathrm{M}$ stage, and TNM stage. The results of GESA showed that the expression of Trim47 influenced NF- $\kappa \mathrm{B}, \mathrm{EMT}$, hypoxia, and apoptosis signaling pathway in GC. Previous studies also had demonstrated that upregulated Trim47 stimulated cell proliferation and metastasis via exerting an inhibitory effect on P53 and a facilitatory effect on the NF- $\mathrm{BB}$ signaling pathway in non-small cell lung carcinoma [22]. Knockdown of Trim47 in breast cancer cells inhibited cell proliferation, migration, and invasion by inhibiting the activation of the PI3k/AKT signaling pathway [24]. Besides, knockdown of Trim47 also inhibited epithelial-tomesenchymal transition (EMT) in breast cancer [24]. Trim47 promoted colorectal cancer development by upregulating the expression of CCL15 and CCR1 via interacting with SMAD4 and enhancing ubiquitylation and degradation of SMAD4 [23]. We further confirmed Trim47 regulates GC through NF- $\kappa \mathrm{B}$, EMT, hypoxia, and apoptosis signaling pathway by western blot. The result indicated that the expression of Trim47 was significantly associated with NF-кB, EMT, hypoxia, and apoptosis signaling pathway.

In conclusion, our study revealed that elevated expression level of Trim47 was significantly associated with poor OS and DFS in GC patients. Moreover, the expression level of Trim47 was associated with clinicopathological features including tumor differentiation, $\mathrm{T}$ stage, $\mathrm{N}$ stage, $\mathrm{M}$ stage, and TNM stage. Our findings suggest that Trim 47 could be a potential new biomarker to evaluate the prognosis of GC patients.

Acknowledgments: This study was supported by the National Natural Science Foundation of China (Grant NO. 81772579). 


\section{References}

[1] SIEGEL RL, MILLER KD, JEMAL A. Cancer statistics, 2018. CA Cancer J Clin 2018; 68: 7-30. https://doi.org/10.3322/ caac. 21442

[2] GLOBAL BURDEN OF DISEASE CANCER COLLABORATION, FITZMAURICE C, AKINYEMIJU TF, AL LAMI FH, ALAM T et al. Global, Regional, and National Cancer Incidence, Mortality, Years of Life Lost, Years Lived With Disability, and Disability-Adjusted Life-Years for 29 Cancer Groups, 1990 to 2016: A Systematic Analysis for the Global Burden of Disease Study. JAMA Oncol 2018; 4: 1553-1568. https://doi.org/10.1001/jamaoncol.2018.2706

[3] SONG Z, WU Y, YANG J, YANG D, FANG X. Progress in the treatment of advanced gastric cancer. Tumour Biol 2017; 39: 1010428317714626. https://doi. org/10.1177/1010428317714626

[4] FERRO A, PELETEIRO B, MALVEZZI M, BOSETTI C, BERTUCCIO $\mathrm{P}$ et al. Worldwide trends in gastric cancer mortality (1980-2011), with predictions to 2015, and incidence by subtype. Eur J Cancer 2014; 50: 1330-1344. https:// doi.org/10.1016/j.ejca.2014.01.029

[5] SAKA M, MORITA S, FUKAGAWA T, KATAI H. Present and future status of gastric cancer surgery. Jpn J Clin Oncol 2011; 41: 307-313. https://doi.org/10.1093/jjco/hyq240

[6] DASSEN AE, DIKKEN JL, VAN DE VELDE CJ, WOUTERS MW, BOSSCHA K et al. Changes in treatment patterns and their influence on long-term survival in patients with stages I-III gastric cancer in The Netherlands. Int J Cancer 2013; 133: 1859-1866. https://doi.org/10.1002/ijc.28192

[7] SUGANO K. Screening of gastric cancer in Asia. Best Pract Res Clin Gastroenterol 2015; 29: 895-905. https://doi. org/10.1016/j.bpg.2015.09.013

[8] VAN CUTSEM E, SAGAERT X, TOPAL B, HAUSTERMANS K, PRENEN H. Gastric cancer. Lancet 2016; 388: 2654-2664. https://doi.org/10.1016/S0140-6736(16)30354-3

[9] REYMOND A, MERONI G, FANTOZZI A, MERLA G, CAIRO $S$ et al. The tripartite motif family identifies cell compartments. EMBO J 2001; 20: 2140-2151. https://doi. org/10.1093/emboj/20.9.2140

[10] TOMAR D, SINGH R. TRIM family proteins: emerging class of RING E3 ligases as regulator of NF-kappaB pathway. Biol Cell 2015; 107: 22-40. https://doi.org/10.1111/ boc. 201400046

[11] WATANABE M, HATAKEYAMA S. TRIM proteins and diseases. J Biochem 2017; 161: 135-144. https://doi.org/10.1093/ $\mathrm{jb} / \mathrm{mvw} 087$

[12] EAMES HL, SALIBA DG, KRAUSGRUBER T, LANFRANCOTTI A, RYZHAKOV G et al. KAP1/TRIM28: an inhibitor of IRF5 function in inflammatory macrophages. Immunobiology 2012; 217: 1315-1324. https://doi.org/10.1016/j.imbio.2012.07.026

[13] HATAKEYAMA S. TRIM proteins and cancer. Nat Rev Cancer 2011; 11: 792-804. https://doi.org/10.1038/nrc3139

[14] HATAKEYAMA S. TRIM Family Proteins: Roles in Autophagy, Immunity, and Carcinogenesis. Trends Biochem Sci 2017; 42: 297-311. https://doi.org/10.1016/j.tibs.2017.01.002
[15] HE T, CUI J, WU Y, SUN X, CHEN N. Knockdown of TRIM66 inhibits cell proliferation, migration and invasion in colorectal cancer through JAK2/STAT3 pathway. Life Sci 2019; 235: 116799. https://doi.org/10.1016/j.lfs.2019.116799

[16] SATO T, OKUMURA F, ARIGA T, HATAKEYAMA S. TRIM6 interacts with Myc and maintains the pluripotency of mouse embryonic stem cells. J Cell Sci 2012; 125: 1544-1555. https://doi.org/10.1242/jcs.095273

[17] TOCCHINI C, KEUSCH JJ, MILLER SB, FINGER S, GUT $\mathrm{H}$ et al. The TRIM-NHL protein LIN-41 controls the onset of developmental plasticity in Caenorhabditis elegans. PLoS Genet 2014; 10: e1004533. https://doi.org/10.1371/journal. pgen. 1004533

[18] VERSTEEG GA, BENKE S, GARCIA-SASTRE A, RAJSBAUM R. InTRIMsic immunity: Positive and negative regulation of immune signaling by tripartite motif proteins. Cytokine Growth Factor Rev 2014; 25: 563-576. https://doi. org/10.1016/j.cytogfr.2014.08.001

[19] ZAMAN MM, NOMURA T, TAKAGI T, OKAMURA T, JIN $\mathrm{W}$ et al. Ubiquitination-deubiquitination by the TRIM27USP7 complex regulates tumor necrosis factor alpha-induced apoptosis. Mol Cell Biol 2013; 33: 4971-4984. https:// doi.org/10.1128/MCB.00465-13

[20] VANDEPUTTE DA, MEIJE CB, VAN DARTEL M, LEENSTRA S, H IJ-K et al. GOA, a novel gene encoding a ring finger B-box coiled-coil protein, is overexpressed in astrocytoma. Biochem Biophys Res Commun 2001; 286: 574-579. https://doi.org/10.1006/bbrc.2001.5431

[21] FUJIMURA T, INOUE S, URANO T, TAKAYAMA K, YAMADA Y et al. Increased Expression of Tripartite Motif (TRIM) 47 Is a Negative Prognostic Predictor in Human Prostate Cancer. Clin Genitourin Cancer 2016; 14: 298-303. https://doi.org/10.1016/j.clgc.2016.01.011

[22] HAN Y, TIAN H, CHEN P, LIN Q. Trim47 overexpression is a poor prognostic factor and contributes to carcinogenesis in non-small cell lung carcinoma. Oncotarget 2017; 8: 22730-22740. https://doi.org/10.18632/oncotarget.15188

[23] LIANG Q, TANG C, TANG M, ZHANG Q, GAO Y et al. Trim47 is up-regulated in colorectal cancer, promoting ubiquitination and degradation of SMAD4. J Exp Clin Cancer Res 2019; 38: 159. https://doi.org/10.1186/s13046-019-1143-x

[24] WANG Y, LIU C, XIE Z, LU H. Knockdown of Trim47 inhibits breast cancer tumorigenesis and progression through the inactivation of PI3K/Akt pathway. Chem Biol Interact 2020; 317: 108960. https://doi.org/10.1016/j.cbi.2020.108960

[25] JI X, BU ZD, YAN Y, LI ZY, WU AW et al. The 8th edition of the American Joint Committee on Cancer tumor-nodemetastasis staging system for gastric cancer is superior to the 7th edition: results from a Chinese mono-institutional study of 1663 patients. Gastric Cancer 2018; 21: 643-652. https:// doi.org/10.1007/s10120-017-0779-5

[26] CHANDRASHEKAR DS, BASHEL B, BALASUBRAMANYA SAH, CREIGHTON CJ, PONCE-RODRIGUEZ I et al. UALCAN: A Portal for Facilitating Tumor Subgroup Gene Expression and Survival Analyses. Neoplasia 2017; 19: 649658. https://doi.org/10.1016/j.neo.2017.05.002 
[27] WANG Q, WEN YG, LI DP, XIA J, ZHOU CZ et al. Upregulated INHBA expression is associated with poor survival in gastric cancer. Med Oncol 2012; 29: 77-83. https://doi. org/10.1007/s12032-010-9766-y

[28] CUI J, CHEN Y, CHOU WC, SUN L, CHEN L et al. An integrated transcriptomic and computational analysis for biomarker identification in gastric cancer. Nucleic Acids Res 2011; 39: 1197-1207. https://doi.org/10.1093/nar/gkq960

[29] CHO JY, LIM JY, CHEONG JH, PARK YY, YOON SL et al. Gene expression signature-based prognostic risk score in gastric cancer. Clin Cancer Res 2011; 17: 1850-1857. https:// doi.org/10.1158/1078-0432.CCR-10-2180

[30] D'ERRICO M, DE RINALDIS E, BLASI MF, VITI V, FALCHETTI $\mathrm{M}$ et al. Genome-wide expression profile of sporadic gastric cancers with microsatellite instability. Eur J Cancer 2009; 45: 461-469. https://doi.org/10.1016/j.ejca.2008.10.032
[31] KIM SY, VOLSKY DJ. PAGE: parametric analysis of gene set enrichment. BMC Bioinformatics 2005; 6: 144. https://doi. org/10.1186/1471-2105-6-144

[32] NING QY, WU JZ, ZANG N, LIANG J, HU YL et al. Key pathways involved in prostate cancer based on gene set enrichment analysis and meta analysis. Genet Mol Res 2011; 10: 3856-3887. https://doi.org/10.4238/2011.December.14.10

[33] VUNJAKM, VERSTEEG GA. TRIM proteins. Curr Biol 2019; 29: R42-R44. https://doi.org/10.1016/j.cub.2018.11.026

[34] HORIE-INOUE K. TRIM proteins as trim tabs for the homoeostasis. J Biochem 2013; 154: 309-312. https://doi. org/10.1093/jb/mvt076

[35] OZATO K, SHIN DM, CHANG TH, MORSE HC 3RD. TRIM family proteins and their emerging roles in innate immunity. Nat Rev Immunol 2008; 8: 849-860. https://doi. org/10.1038/nri2413 